\title{
New particle searches
}

V. Ruhlmann-Kleider

$D S M / D A P N I A / S P P$, Saclay,

91191 Gif-sur-Yvette Cedex, FRANCE

\section{Introduction}

This review covers a few selected topics from the searches performed at Tevatron, HERA and LEP2. Details on the data samples analysed at the time of the conference are given in Table 1 .

\begin{tabular}{|c|c|c|c|c|c|}
\hline collider & experiments & beams & $\sqrt{s}$ & period & $\begin{array}{c}\mathcal{L} / \text { expt } \\
\left(\mathrm{pb}^{-1}\right)\end{array}$ \\
\hline Tevatron & CDF/D0 & $\mathbf{p} \overline{\mathbf{p}}$ & $1.8 \mathrm{TeV}$ & $' 87 / ' 96$ & $\sim 110$ \\
HERA & H1/ZEUS & $\mathbf{e}^{+} \mathbf{p}$ & $300 \mathrm{GeV}$ & $' 94 / ' 97$ & $\sim 40$ \\
& & $\mathbf{e}^{-} \mathbf{p}$ & $318 \mathrm{GeV}$ & $' 98 / ' 99$ & $\sim 15$ \\
LEP2 & ALEPH/DELPHI & $\mathbf{e}^{+} \mathbf{e}^{-}$ & 130 to $183 \mathrm{GeV}$ & $' 95 / ' 97$ & $\sim 90$ \\
& L3/OPAL & $\mathbf{e}^{+} \mathbf{e}^{-}$ & $189 \mathrm{GeV}$ & $' 98$ & $\sim 170$ \\
& & $\mathbf{e}^{+} \mathbf{e}^{-}$ & $192 / 196 \mathrm{GeV}$ & $' 99$ & $\sim 105$ \\
\hline
\end{tabular}

Table 1: Data samples analysed for the '99 summer conferences by experiments at Tevatron, HERA and LEP2. The last column gives the integrated luminosity per experiment for each of the data taking periods.

New particle searches are dominated by LEP whose results come mostly from data up to $189 \mathrm{GeV}$ except for some updates from '99 data up to $196 \mathrm{GeV}$. Combined results from the LEP experiments also exist on some subjects and are denoted ADLO.

After a brief outline of the searches on exotic particles, results on supersymmetric particles and Higgs bosons are detailed. All exclusion limits are at the 95\% Confidence Level.

\section{$2 \quad$ Exotic particles}

Searches for exotic particles encompass a great variety of topics, such as technicolor particles [1], new Z' bosons [2, 3], four fermion contact interactions [4, 3], new, excited 
or exotic fermions [5], leptoquarks. Constraints are derived at the three colliders either from direct searches or from comparing precise measurements with Standard Model (SM) expectations.

As an illustration, the case of leptoquark (LQ) searches is detailed. The phenomenology of leptoquarks is describbed by three parameters: $\mathrm{M}_{\mathrm{LQ}}$, the LQ mass, $\lambda_{\text {lq }}$ and $\beta_{1}$, the LQ coupling and branching ratio into a given pair of SM lepton and quark, $(1, q)$. Results are interpreted either assuming leptoquarks to be coupled to a single SM generation, with fixed $\beta_{1}$ (as in the BRW model [6]) or in more generic models, with $\beta_{1}$ variable and possible mixed couplings.

\begin{tabular}{|c|c|c|c|c|}
\hline collider & LQ type & assumptions & limit $\left(\mathrm{GeV} / c^{2}\right)$ & couplings \\
\hline Tevatron & 1st gen. LQ & $\beta_{\mathrm{e}}=1$ & 242 & any $\lambda_{\text {eq }}$ \\
Tevatron & 2nd gen. LQ & $\beta_{\mu}=1$ & 202 & any $\lambda_{\mu \mathrm{q}}$ \\
Tevatron & 3rd gen. LQ & $\beta_{\nu_{\tau}}=1$ & 149 & any $\lambda_{\nu_{\tau} \mathrm{q}}$ \\
Tevatron & 3rd gen. LQ & $\beta_{\tau}=1$ & 99 & any $\lambda_{\tau \mathrm{q}}$ \\
HERA & 1st gen. LQ & BRW model & 265 & $\lambda_{\text {eq }}=0.3$ \\
LEP2 & 1st or 2nd gen. $\mathrm{S}_{1, L}$ & BRW model & 370 & $\lambda_{\mathrm{lq}}=0.3$ \\
\hline
\end{tabular}

Table 2: Limits on leptoquarks from Tevatron, HERA and LEP2. Specific values have been assumed for the LQ branching ratios and/or couplings to SM particles. Precise measurement at LEP2 allow to probe high masses in some specific cases only.

The current constraints in the first approach [7, 8] are summarized in Table 2. The interpretation in generic models has been pioneered by H1 [7]. As an example, Figure 11 shows the regions excluded by $\mathrm{H} 1$ and DO in the plane $\mathrm{M}_{\mathrm{LQ}}$ vs $\beta_{\mathrm{e}}$ for first generation leptoquarks. HERA sensitivity extends down to small $\beta_{\mathrm{e}}$ even for small couplings, e.g. for $\beta_{\mathrm{e}} \sim 10 \%$ and $\lambda_{\mathrm{eq}} \sim 0.05$ leptoquark masses up to $200 \mathrm{GeV} / \mathrm{c}^{2}$ are excluded. The interesting case of mixed couplings has also been studied [7]. To cite one result, leptoquarks with couplings to both (e, q) and $(\tau, q)$ have been found to be excluded with a sensitivity similar to that quoted for pure first generation leptoquarks.

\section{$3 \quad$ Supersymmetric particles}

All supersymmetric particle searches are conducted within the Minimal Supersymmetric Standard Model (MSSM) with additional assumptions to decrease the number of free parameters. Depending on those assumptions, the phenomenology differs and so do the experimental signatures. At present, three theoretical frameworks are studied. 


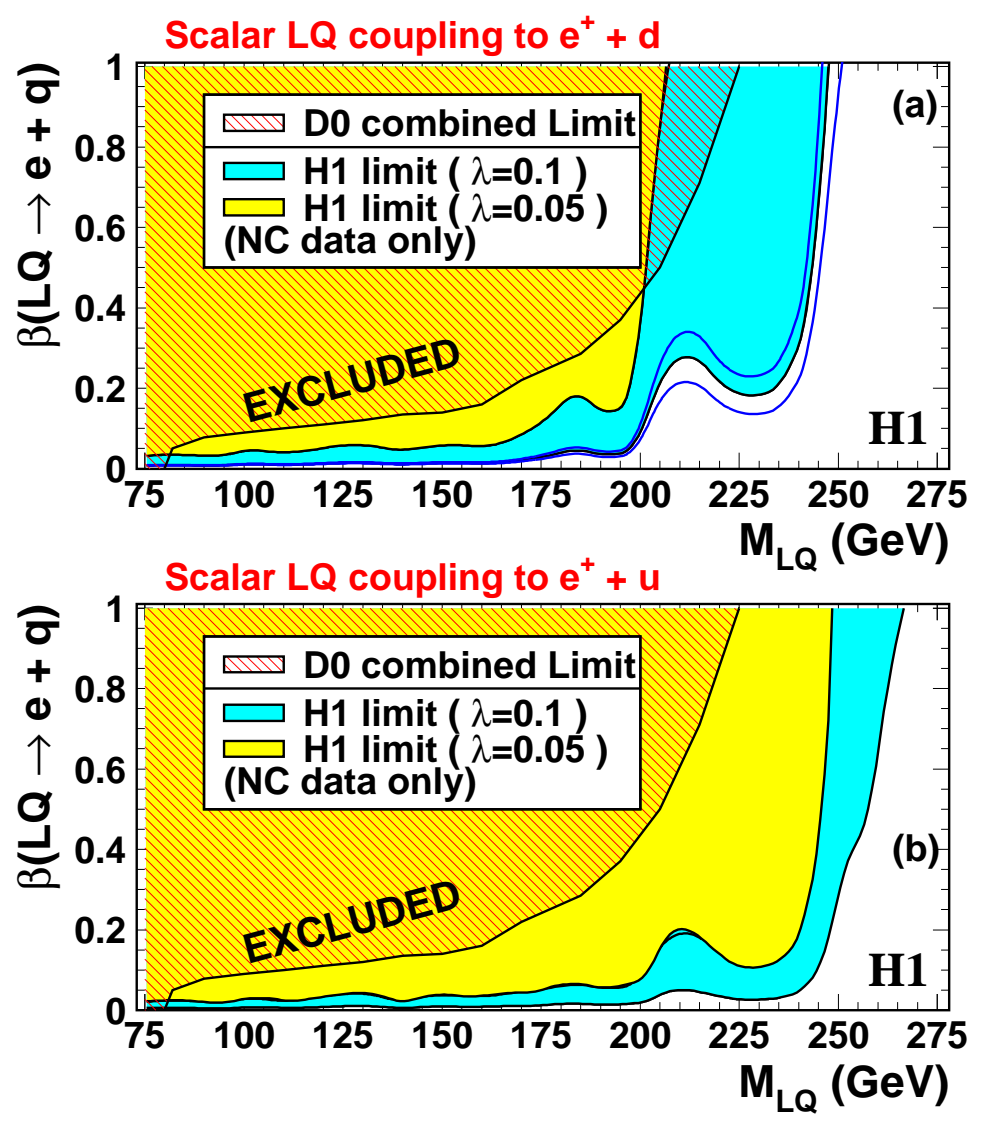

Figure 1: Constraints on first generation leptoquarks from $\mathrm{H} 1$ and DO. For $\lambda=0.1$, the error bands illustrate the sensitivity to $\mathrm{d}$ and $\mathrm{u}$ quark densities.

\subsection{Constrained MSSM}

The most common framework assumes R-parity conservation and soft supersymmetry breaking mediated by gravity. Soft breaking terms are thus unified at high energy (the so-called GUT scale) and the number of free parameters is reduced to five: the common sfermion' mass term at GUT scale, $\mathrm{m}_{0}$, the common gaugino mass term at GUT scale, $\mathrm{m}_{1 / 2}$, the common trilinear coupling at GUT scale, $\mathrm{A}_{0}$, the Higgs mixing parameter, $\mu$, and the ratio of the two Higgs doublet vacuum expectation values,

\footnotetext{
${ }^{1}$ Tevatron experiments use a somewhat different and more constrained scheme, called minimal supergravity (mSUGRA), which defines $\mathrm{m}_{0}$ as a common scalar mass term at the GUT scale and assumes in addition radiative EW symmetry breaking, so that $\mu$ is fixed up to a sign.
} 
$\tan \beta$.

The phenomenology at low energy is derived using renormalisation group equations. The lightest supersymmetric particle (LSP) is in most cases the lightest neutralino, $\widetilde{\chi}_{1}^{0}$. Due to R-parity conservation, sparticles are produced in pairs and decay in their SM partner and a sparticle. At the end of the decay chain, the LSP appears and, as it is stable, gives rise to missing energy. Results on all types of sparticles have been reported at the conference.

\subsubsection{Sfermions}

Charged sleptons and light squarks are searched for at LEP through the decays summarized in Table 3, which are the dominant ones if the researched sparticle is the next lightest sparticle. The experimental sensitivity depends on both the sfermion mass and the mass difference $\Delta M$ between the sfermion and the LSP. The experimental sensitivity starts from $\Delta M$ above a few $\mathrm{GeV} / c^{2}$ and covers sfermion masses up to 70 to $90 \mathrm{GeV} / c^{2}$ depending on the sfermion, as can be seen in Table 3 for the LEP combination at $189 \mathrm{GeV}$ [9]. Similar sensitivities are reached by the individual LEP experiments at $196 \mathrm{GeV}$ [10]. All LEP results are derived for minimal production cross-sections and hence have a general validity.

\begin{tabular}{|c|c|c|c|c|c|}
\hline experiment & sfermion & decay & $\begin{array}{c}\text { obs. limit } \\
\left(\mathrm{GeV} / c^{2}\right)\end{array}$ & $\begin{array}{c}\text { exp. limit } \\
\left(\mathrm{GeV} / c^{2}\right)\end{array}$ & $\begin{array}{c}\Delta M \\
\left(\mathrm{GeV} / c^{2}\right)\end{array}$ \\
\hline $\mathrm{ADLO}$ & $\widetilde{\mathrm{e}}_{\mathrm{R}}$ & e $\widetilde{\chi}_{1}^{0}$ & 89 & 90 & $>15$ \\
$189 \mathrm{GeV}$ & $\widetilde{\mu}_{\mathrm{R}}$ & $\mu \widetilde{\chi}_{1}^{0}$ & 84 & 83 & $>15$ \\
& $\widetilde{\tau}_{\mathrm{R}}$ & $\tau \widetilde{\chi}_{1}^{0}$ & 71 & 77 & $>15$ \\
\hline $\mathrm{ADLO}$ & $\widetilde{\mathrm{t}}_{1}$ & $\mathrm{c} \widetilde{\chi}_{1}^{0}$ & 87 & 84 & $>10$ \\
$189 \mathrm{GeV}$ & $\widetilde{\mathrm{t}}_{1}$ & b l $\widetilde{\nu}$ & 90 & 87 & $>10$ \\
& $\widetilde{\mathrm{b}}_{1}$ & $\mathrm{~b} \widetilde{\chi}_{1}^{0}$ & 80 & 68 & $>10$ \\
\hline $\mathrm{CDF}$ & $\mathrm{t}_{1}$ & $\mathrm{c} \widetilde{\chi}_{1}^{0}$ & 89 & - & $>40$ \\
run I & $\widetilde{\mathrm{b}}_{1}$ & b $\widetilde{\chi}_{1}^{0}$ & 105 & - & $>40$ \\
\hline
\end{tabular}

Table 3: Lower limits on sfermion masses. Combined LEP results up to $189 \mathrm{GeV}$ are given as well as CDF results from run I. The LEP expected limits are computed from simulation only, assuming no signal. The last column gives the range of validity of the limits expressed as a minimal difference between the masses of the sfermion and the $\operatorname{LSP}\left(\widetilde{\chi}_{1}^{0}\right.$ or $\left.\widetilde{\nu}\right)$.

Light squarks are also searched for at Tevatron [11]. The experimental sensitivity is complementary from that of LEP since it covers higher squark masses and starts at higher $\Delta M$, as illustrated in Table 3. Searches for heavy squarks and gluinos 
belong to Tevatron [12]. The final states result from $\widetilde{\mathrm{q}}$ and $\widetilde{\mathrm{g}}$ cascade decays to the LSP and quarks, gluons, $\mathrm{W}$ or $\mathrm{Z}$ bosons. The present experimental reach is around $250 \mathrm{GeV} / c^{2}$ but it must be noted that most results are derived for specific values of some mSUGRA parameters $\left(\mathrm{A}_{0}=0, \mu<0\right.$ and $\left.\tan \beta=2\right)$ which restricts their range of validity.

\subsubsection{Charginos and neutralinos}

Due to its excellent coverage of the various signatures of supersymmetry, LEP provides limits on the masses of the lightest chargino and neutralino which are practically absolute in the constrained MSSM framework.

Direct searches for the lightest chargino $\tilde{\chi}_{1}^{ \pm}$provide limits on $m_{\widetilde{\chi}_{1}^{ \pm}}$close to the kinematical limit in most of the parameter space [10]. As $\widetilde{\chi}_{1}^{0}$ cannot be detected, direct neutralinos searches rely on the production of heavier neutralinos $\left(\right.$ e.g. $\widetilde{\chi}_{1}^{0} \widetilde{\chi}_{2}^{0}$ or $\left.\tilde{\chi}_{2}^{0} \widetilde{\chi}_{2}^{0}\right)$ and thus bring little constraint on $m_{\widetilde{\chi}_{1}^{0}}$ except when $\tan \beta$ is close to 1 . But, combining the results from $\tilde{\chi}_{i}^{0}$ and $\tilde{\chi}_{1}^{ \pm}$searches provides a limit on $m_{\widetilde{\chi}_{1}^{0}}$ valid for large $m_{0}$ whatever the other parameters [10].

For low values of $m_{0}$ (that is light $\widetilde{\nu}$ ), the $\widetilde{\chi}_{1}^{ \pm}$production cross-section drops due to the negative interference between the s-channel production process and the t-channel $\widetilde{\nu}$ exchange diagram. The $\widetilde{\chi}_{1}^{ \pm}$decay into $l \widetilde{\nu}$ becomes dominant and escapes detection when $\widetilde{\chi}_{1}^{ \pm}$and $\widetilde{\nu}$ are denegerated. Under the same conditions, the $\widetilde{\chi}_{i}^{0}$ production increases (because of a positive interference term) but the $\widetilde{\chi}_{\mathrm{i}}^{0}$ decay into $\nu \widetilde{\nu}$ opens, leading to invisible final states. But, low values of $\mathrm{m}_{0}$ also mean light sleptons, which are thus within experimental reach. Combining $\widetilde{\chi}_{\dot{i}}^{0}, \widetilde{\chi}_{1}^{ \pm}$and $\widetilde{1}$ searches, limits on $m_{\widetilde{\chi}_{1}^{ \pm}}$and $m_{\widetilde{\chi}_{1}^{0}}$ valid for $\mathrm{A}_{0}=0$ and for all values of the other parameters can be derived [13, 14, as illustrated in Table 4 and Figure 2. Preliminary studies varying $\mathrm{A}_{0}$ (that is allowing for $\widetilde{\tau}$ mixing) show that the limit on $m_{\widetilde{\chi}_{1}^{ \pm}}$may be affected but not that on $\widetilde{\chi}_{1}^{0}[14$.

\begin{tabular}{|c|c|c|c|c|}
\hline experiment & $\begin{array}{c}\sqrt{s} \\
(\mathrm{GeV})\end{array}$ & $\begin{array}{c}\text { searches } \\
\text { combined }\end{array}$ & $\begin{array}{c}\text { limit } \\
\left(\mathrm{GeV} / c^{2}\right)\end{array}$ & validity \\
\hline OPAL & 196 & $\widetilde{\chi}_{1}^{ \pm}$ & $m_{\widetilde{\chi}_{1}^{ \pm}}>97.6$ & large $\mathrm{m}_{0}, \Delta M>10 \mathrm{GeV} / c^{2}$ \\
ALEPH & 196 & $\widetilde{\chi}_{1}^{ \pm}, \widetilde{\chi}_{\mathrm{i}}^{0}$ & $m_{\widetilde{\chi}_{1}^{0}}>34.0$ & large $\mathrm{m}_{0}$ \\
L3 & 189 & $\widetilde{\chi}_{1}^{ \pm}, \widetilde{\chi}_{\mathrm{i}}^{0}, \widetilde{l}$ & $m_{\widetilde{\chi}_{1}^{ \pm}}>67.7$ & $\mathrm{~A}_{0}=0$ \\
L3 & 189 & $\widetilde{\chi}_{1}^{ \pm}, \widetilde{\chi}_{\mathrm{i}}^{0}, \widetilde{l}$ & $m_{\widetilde{\chi}_{1}^{0}}>32.5$ & absolute \\
\hline
\end{tabular}

Table 4: Lower limits on $m_{\widetilde{\chi}_{1}^{ \pm}}$and $m_{\widetilde{\chi}_{1}^{0}}$, from LEP. As more results are used, the range of validity of the limits extends . 

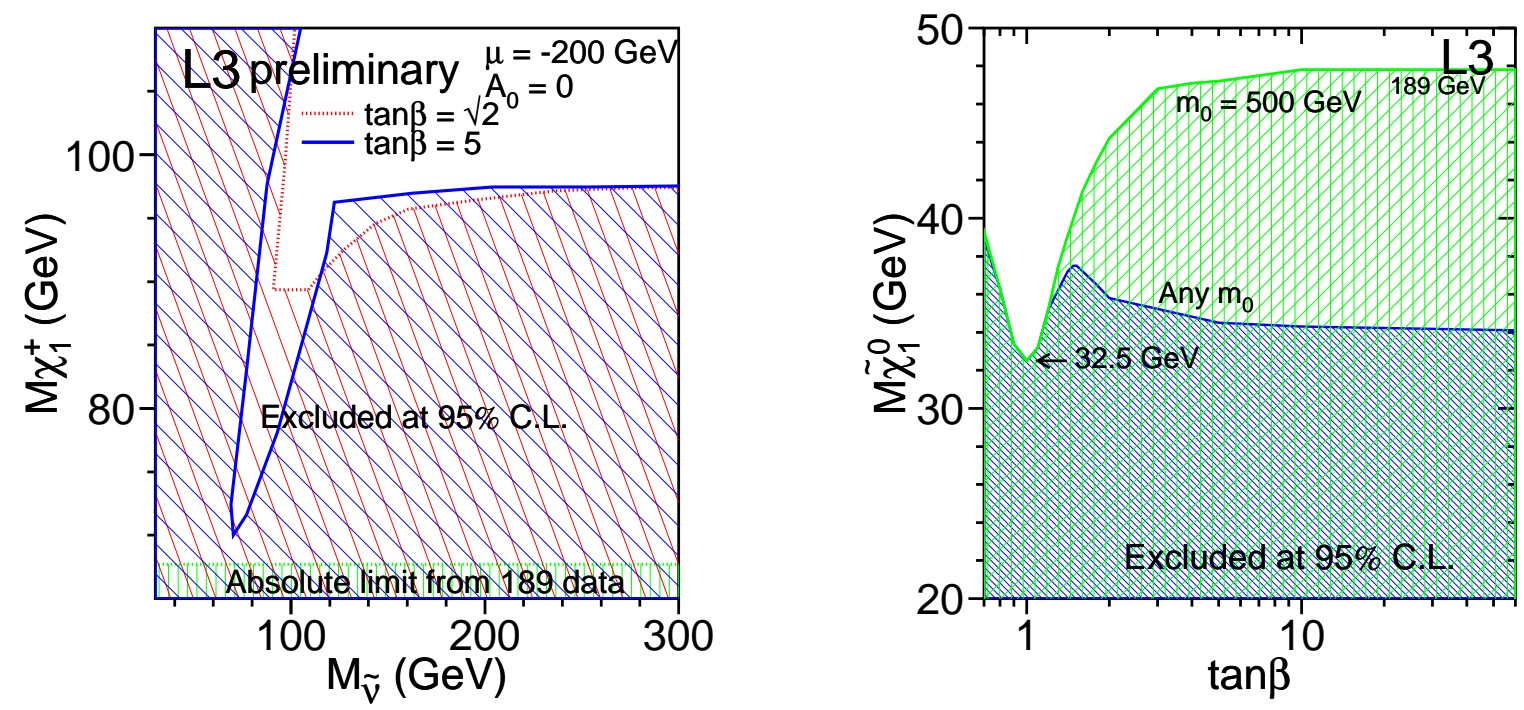

Figure 2: Left: L3 Limit on $m_{\widetilde{\chi}_{1}^{ \pm}}$as a function of $m_{\widetilde{\nu}}$. The absolute limit from data up to $189 \mathrm{GeV}$ is compared with the '99 limits obtained for two sets of the MSSM parameters. For a heavy $\widetilde{\nu}$ the kinematical limit is almost reached while for a $\widetilde{\nu}$ around $70 \mathrm{GeV} / c^{2}$, the limits drop. Right: L3 limit on $m_{\widetilde{\chi}_{1}^{0}}$ as a function of $\tan \beta$ from data up to $189 \mathrm{GeV}$. The limit obtained at large $\mathrm{m}_{0}$ whatever the other parameters is compared with the limit obtained when $\mathrm{m}_{0}$ is also allowed to vary. The absolute limit corresponds to $\tan \beta=1$.

\section{$3.2 \quad$ R-parity breaking}

In the second theoretical framework, soft supersymmetry breaking is still mediated by gravity but R-parity $\left(R_{p}\right)$ is assumed to be broken via an extra-term to the superpotential of the form:

$$
\mathcal{W}=\lambda_{i j k} \mathrm{~L}_{i} \mathrm{~L}_{j} \overline{\mathrm{E}}_{k}+\lambda_{i j k}^{\prime} \mathrm{L}_{i} \mathrm{Q}_{j} \overline{\mathrm{D}}_{k}+\lambda_{i j k}^{\prime \prime} \overline{\mathrm{U}}_{i} \overline{\mathrm{D}}_{j} \overline{\mathrm{D}}_{k}
$$

where $i j k$ denote generation indices and the capital letters refer to superfields associated to left-handed doublets of leptons (L) and quarks (Q), and right-handed singlets of charged leptons (E), down-type quarks (D) and up-type quarks (U). $\mathcal{W}$ implies violation of the leptonic and baryonic numbers. In addition to the five parameters related to supersymmetry breaking $\left(\mathrm{m}_{0}, \mathrm{~m}_{1 / 2}, \mathrm{~A}_{0}, \mu\right.$ and $\left.\tan \beta\right), \mathrm{R}_{\mathrm{p}}$ breaking $\left(\mathrm{B}_{\mathrm{p}}\right.$ ) introduces 45 couplings $\left(9 \lambda_{i j k}, 27 \lambda_{i j k}^{\prime}\right.$ and $\left.9 \lambda_{i j k}^{\prime \prime}\right)$. For sake of simplicity, searches are conducted assuming only one coupling to dominate at a time and all sparticles to decay close to the interaction vertex. This latter hypothesis corresponds to assuming the $\mathrm{B}_{\mathrm{p}}$ couplings to be greater than values which are at least two orders of magnitude 
below the current experimental limits on most couplings.

Compared to $\mathrm{R}_{\mathrm{p}}$ conservation, $\mathrm{B}_{\mathrm{p}}$ modifies the phenomenology at low energy. Single sparticle production is possible, the LSP (which is still $\tilde{\chi}_{1}^{0}$ in most cases) is no longer stable and the sparticle decay patterns change a lot. Sparticle can decay into SM particles through one $\mathrm{B}_{\mathrm{p}}$ vertex (e.g. $\widetilde{\nu}$ case in Figure $3 \mathrm{a}$ ) or via an $\mathrm{R}_{\mathrm{p}}$ conserving vertex leading to an off-shell sparticle that decays through an $\mathrm{B}_{\mathrm{p}}$ vertex $\left(e . g \cdot \widetilde{\chi}_{1}^{0}\right.$ and $\tilde{\chi}_{1}^{ \pm}$cases in Figure $3 \mathrm{a}$ ). These decays are referred to as direct decays. A second type of decays, called indirect decays, implies cascade decays to SM particles through several $R_{p}$ conserving and $R_{p}$ vertices with some sparticles on-shell, such as in Figure §b.

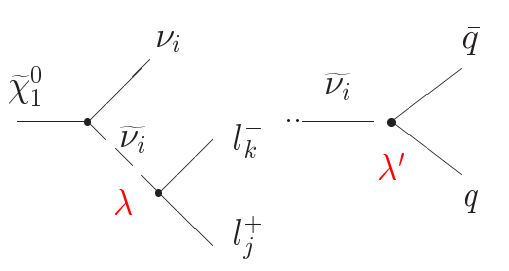

a) direct decays

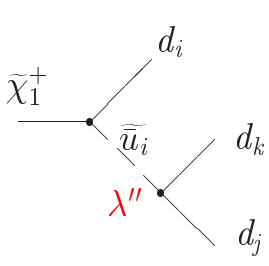

Figure 3: Examples of decays allowed by $\mathrm{R}_{\mathrm{p}}$ violation.

Thus, there are many final states to consider, with multileptons and/or multijets and possibly missing energy from neutrinos. As in the constrained MSSM case, all types of sparticles have been searched for.

\subsubsection{Charginos and neutralinos}

As in the $R_{p}$ conserving scheme, LEP sets constraints on charginos and neutralinos which are valid over wide ranges of the underlying parameters (except $A_{0}$ which is set to 0 ). The $\widetilde{\chi}_{1}^{ \pm}$and $\widetilde{\chi}_{\mathrm{i}}^{0}$ production modes are as in the $\mathrm{R}_{\mathrm{p}}$ conserving case while their decay pattern is completely modified. The most notable change concerns the LSP whose production leads to observable final states whatever the dominant $\mathrm{B}_{\mathrm{p}}$ coupling. All experimental signatures expected from the production and decays of $\widetilde{\chi}_{1}^{+} \tilde{\chi}_{1}^{-}, \widetilde{\chi}_{1}^{0}$ $\widetilde{\chi}_{1}^{0}$ and $\widetilde{\chi}_{2}^{0} \widetilde{\chi}_{1}^{0}$ have been investigated at LEP2. Due to $\widetilde{\chi}_{1}^{0}$ being detectable, combining $\widetilde{\chi}_{\mathrm{i}}^{0}$ and $\widetilde{\chi}_{1}^{ \pm}$searches alone suffices to derive constraints [15] on the sparticle masses valid for $A_{0}=0$ and all values of the other parameters, as illustrated in Table 5 .

\subsubsection{Sneutrinos}

Another appreciable change due to $\mathrm{B} / \mathrm{p}$ is the allowed decay of sneutrinos which make them directly observable e.g. at LEP through double and single productions. 


\begin{tabular}{|c|c|c|c|}
\hline $\mathrm{B}_{\mathrm{p}}$ coupling & \multicolumn{3}{|c|}{ limits $\left(\mathrm{GeV} / c^{2}\right)$} \\
type & $\mathrm{M}_{\widetilde{\chi}_{1}^{0}}$ & $\mathrm{M}_{\widetilde{\chi}_{2}^{0}}$ & $\mathrm{M}_{\widetilde{\chi}_{1}^{ \pm}}$ \\
\hline$\lambda, \lambda^{\prime}$ couplings: & 30 & 50 & 94 \\
\hline$\lambda^{\prime \prime}$ couplings: & 32 & 67 & 94 \\
\hline
\end{tabular}

Table 5: L3 limits on $\widetilde{\chi}_{1}^{0}, \widetilde{\chi}_{2}^{0}$ and $\widetilde{\chi}_{1}^{ \pm}$masses in the $\mathrm{B}_{\mathrm{p}}$ scheme, from data up to $189 \mathrm{GeV}$.

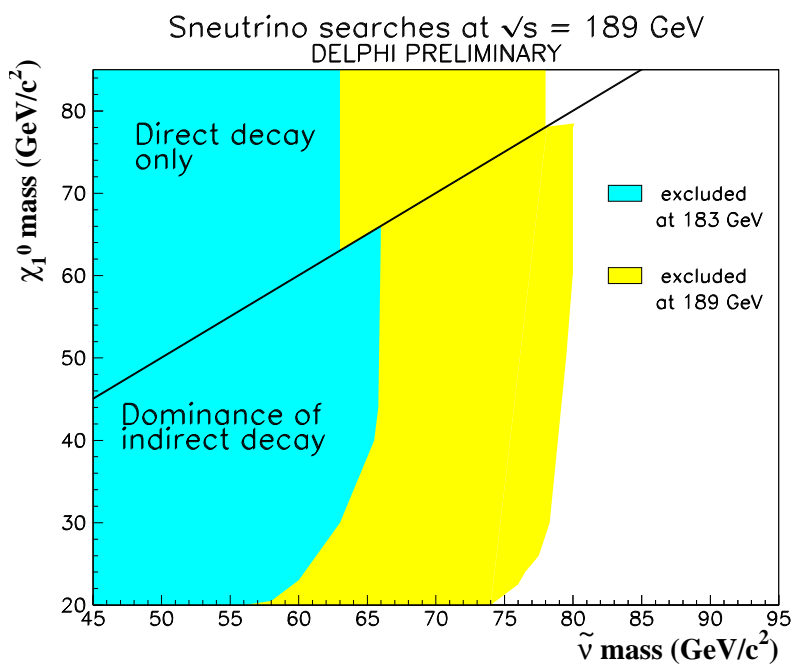

Figure 4: DELPHI limit on sneutrino pair production as allowed by $\mathrm{B}_{\mathrm{p}}$ with a dominant $\lambda_{133}$ coupling.

All final states expected from pair production have been searched for. An example of result for a dominant $\lambda_{133}$ coupling is given in Figure 4 . Using also the limit on $m_{\widetilde{\chi}_{1}^{0}}$, exclusion limits can be derived for each of the three $\mathrm{B}_{\mathrm{p}}$ coupling classes. The current limits [15] are presented in Table 6. They are valid for all values of the underlying parameters, except $\mathrm{A}_{0}$ which is set to 0 .

Single production of $\widetilde{\nu}_{\mu}$ or $\widetilde{\nu}_{\tau}$ sneutrinos is also possible at LEP, as illustrated in Figure 5. Sneutrino direct decays would lead to effects observable as deviations wrt SM expectations while indirect decays would manifest as specific final states that require dedicated searches. In both cases, constraints on sneutrino masses have been derived as a function of the $\mathrm{B}_{\mathrm{p}}$ couplings. Currently, masses between 100 to $200 \mathrm{GeV} / c^{2}$ are probed and limits on couplings are of the order of a few $10^{-2}$ [16]. 


\begin{tabular}{|c|c|c|c|}
\hline $\begin{array}{c}\mathrm{B}_{\mathrm{p}} \text { coupling } \\
\text { type }\end{array}$ & experiment & $\begin{array}{c}\text { limit on } m_{\widetilde{\nu}} \\
\left(\mathrm{GeV} / c^{2}\right)\end{array}$ & $\widetilde{\nu}$ flavour \\
\hline$\lambda$ couplings: & DELPHI & 78 & any $\widetilde{\nu}$ flavour \\
$\lambda^{\prime}$ couplings: & ALEPH & 56 & any $\widetilde{\nu}$ flavour \\
$\lambda^{\prime \prime}$ couplings: & ALEPH & 77 & $\widetilde{\nu}_{\mathrm{e}}$ only \\
\hline
\end{tabular}

Table 6: LEP limits on $\widetilde{\nu}$ masses in the $\mathrm{B}_{\mathrm{p}}$ scheme, from data up to $189 \mathrm{GeV}$.

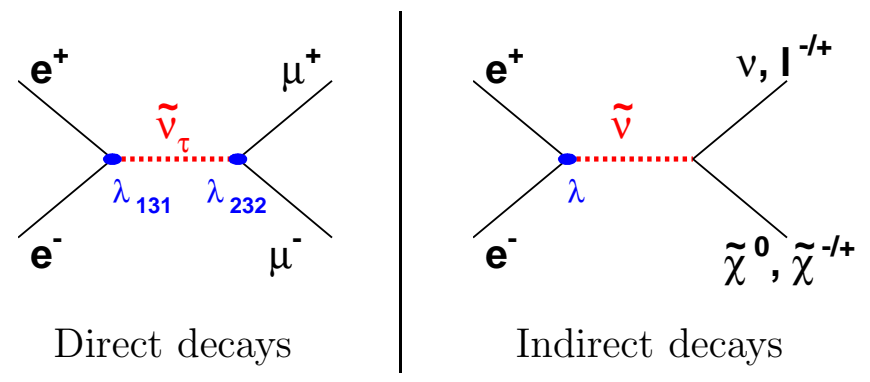

Figure 5: Single production of sneutrinos at LEP as allowed by $\mathrm{B}_{\mathrm{p}}$.

\subsubsection{Charged sleptons and squarks}

Charged sleptons and light squarks are searched for at LEP. The coverage of the final states expected in case of $\mathrm{B}_{\mathrm{p}}$ (from direct and indirect decays and for any type of couplings) is not yet as complete as for the other sparticles. The results achieved so far [15] are summarized in Table 7. As for other LEP results, they hold for $\mathrm{A}_{0}=0$, irrespective of the values of the other underlying parameters.

Finally, there are also constraints on heavy squarks and gluinos if $R_{p}$ is violated. LEP experiments reinterpret their results about leptoquark searches [17] while dedicated searches are performed at HERA and Tevatron for particular $\mathrm{B}_{\mathrm{p}}$ couplings. HERA experiments have access mostly to $\lambda_{1 j 1}^{\prime}$ couplings and derive constraints on heavy squark masses as a fuction of $\lambda_{1 j 1}^{\prime}$, varying the MSSM underlying parameters. Currently, masses around $240 \mathrm{GeV} / c^{2}$ are excluded for $\lambda_{1 j 1}^{\prime} \sim 0.3$ [18. Using multilepton events in run I data, Tevatron experiments have probed $\lambda_{1 j k}^{\prime}$ and $\lambda_{121}$ couplings, and constrained gluino and heavy squark masses. The limits achieved are around 250 (resp. 350) $\mathrm{GeV} / c^{2}$ for the $\lambda_{1 j k}^{\prime}\left(\right.$ resp. $\lambda_{121}$ ) coupling analysis [19]. These limits are valid for $\mathrm{A}_{0}=0, \mu<0$ and $\tan \beta=2$. Most of the other choices (in particular, higher values of $\tan \beta$ or $\mu>0$ ) would lead to lower limits, due to a loss in sensitivity (resulting from reduced branching fractions into leptons, softer leptons...) [19. 


\begin{tabular}{|c|c|c|c|}
\hline $\begin{array}{c}\mathrm{B}_{\mathrm{p}} \text { coupling } \\
\text { type }\end{array}$ & experiment & sparticle & $\begin{array}{c}\text { mass limit } \\
\left(\mathrm{GeV} / c^{2}\right)\end{array}$ \\
\hline$\lambda$ couplings: & ALEPH & $\widetilde{\mathrm{e}}_{\mathrm{R}}$ & 84 \\
$\lambda$ couplings: & ALEPH & $\widetilde{\mu}_{\mathrm{R}}, \widetilde{\tau}_{\mathrm{R}}$ & 60 \\
$\lambda^{\prime}$ couplings: & OPAL & $\widetilde{\mathrm{t}}_{1}$ & 84 \\
$\lambda^{\prime \prime}$ couplings: & OPAL & $\widetilde{\mathrm{t}}_{1}$ & 79 \\
\hline
\end{tabular}

Table 7: LEP limits on charged slepton and light squark masses in the $\mathrm{B}_{\mathrm{p}}$ scheme, from data up to $189 \mathrm{GeV}$. The $\widetilde{\mathrm{t}}_{1}$ limits are valid for any value of the stop mixing angle.

\subsection{Gauge mediated supersymmetry breaking}

The third theoretical framework assumes $R_{p}$ conservation and soft supersymmetry breaking mediated by gauge interactions. Such models 20] usually need six basic parameters: the supersymmetry breaking scale, $\sqrt{\mathrm{F}}$, the universal mass scale of supersymmetric particles, $\Lambda$, the messenger mass scale, $M_{s}$, the number of messenger generations, $\mathrm{n}_{\mathrm{s}}$, the Higgs mixing parameter, $\mu$, and the ratio of the two Higgs doublet vacuum expectation values, $\tan \beta$. The breaking scale is expected to be much lower than in gravity-mediated models, down to about $10^{4} \mathrm{GeV}$.

As far as phenomenology at low energy is concerned, $R_{p}$ conservation implies as usual sparticle pair production and a stable LSP. As a consequence of gauge-mediated breaking, the LSP is the gravitino, $\widetilde{G}$, whose mass depends on $\sqrt{\mathrm{F}}$ and thus is expected to be very small, in the range $\left[10^{-6} \mathrm{eV}, 1 \mathrm{keV}\right]$. The next lightest sparticle (NSLP) is either $\widetilde{\chi}_{1}^{0}$ or a charged slepton $\left(\widetilde{\tau}_{1}\right.$ or three degenerated $\left.\widetilde{l}\right)$. The NSLP lifetime is governed by the $\widetilde{G}$ mass and hence can be non negligible, giving rise to specific topologies, some being experimental challenges.

\subsection{1 $\tilde{\chi}_{1}^{0}$ NLSP}

The main decay of a $\tilde{\chi}_{1}^{0}$ NLSP would be in $\gamma \widetilde{\mathrm{G}}$. Thus $\tilde{\chi}_{1}^{0}$ searches rely on final states with photons, either single-photon or diphoton events. Such final states provide clean experimental signatures and have been used since long to chase new physics, whatever the underlying theoretical framework. Results are usually expressed as model-independent upper limits on the cross-section times branching fraction product, as a function of the mass of the unknown particle decaying to a photon plus missing energy. These cross-section limits are then compared with predictions from various models 21, 22], such as the GMSB scenario explaining an ee $\gamma \gamma$ event reported by CDF some years ago. To quote but one result, even if not strictly a GSMB model, cross-section limits have been converted into a lower limit on the mass of a superlight 
$\widetilde{\mathrm{G}}$, assuming all other sparticles to be above threshold. The current limit from singlephoton events at LEP2 [21] is $10^{-5} \mathrm{eV} / c^{2}$, similar to the result reached by CDF using monojet events [23].

Topologies more specific to GMSB models have also been searched for. The first example is given by $\tilde{\chi}_{1}^{0}$ searches in case of long neutralino lifetimes. The decay photons would not be produced at the interaction point but could have large impact parameters. Searches for non-pointing single-photon events [21] cover that case. Searches for sparticles heavier than $\widetilde{\chi}_{1}^{0}$ have also been performed, so far for charginos and sleptons [24] only. Compared with the topologies expected in the gravity-mediated scheme, the final states are identical except for additional photons that can be detected if the NLSP lifetime is not too long. As photons help to better discriminate against background, the exclusion limits for negligible or moderate NSLP lifetimes are usually tigther than those in the gravity-mediated framework.

\subsection{2 $\widetilde{\tau}_{1}$ NLSP}

The main decay of a $\widetilde{\tau}_{1}$ NLSP would be in $\tau \widetilde{\mathrm{G}}$, thus giving the same experimental signature as in the gravity-mediated case only if the $\widetilde{\tau}_{1}$ lifetime is negligible. In the contrary case, $\widetilde{\tau}_{1}$ decays would lead to kinks, large impact parameters or decay vertices. Eventually, a $\widetilde{\tau}_{1}$ decaying outside the detector would appear as a stable charged particle. All these experimental signatures have been used in $\widetilde{\tau}_{1}$ searches [24]. An example of result is given in Figure 6 which illustrates the interplay of the different signatures as a function of the $\widetilde{G}$ mass which defines the $\widetilde{\tau}_{1}$ mean decay length. Irrespective of the $\widetilde{\mathrm{G}}$ mass, these results exclude a $\widetilde{\tau}_{1}$ NLSP up to $73 \mathrm{GeV} / c^{2}$. The limit is $6 \mathrm{GeV} / c^{2}$ higher if the results are reinterpreted in a scenario with three degenerated co-NLSP charged sleptons.

Searches for sparticles heavier than $\widetilde{\tau}_{1}$ have also been performed, so far for charginos, neutralinos and sleptons [24] and only for negligible NLSP lifetimes.

\subsubsection{Constraining underlying parameters}

Even if not complete, the coverage of the final states expected from GMSB models is at present sufficient to exclude large fractions of the parameter space in order to set constraints on the key parameters of the model, like the NLSP mass or $\Lambda$. A first attempt has been reported in [25] for a minimal GMSB model.

\subsection{Other searches, recent developments}

To conclude about supersymmetry, it is worth mentioning a few complementary results from LEP, either recent ones or results aside the main stream of searches described in the previous sections. 


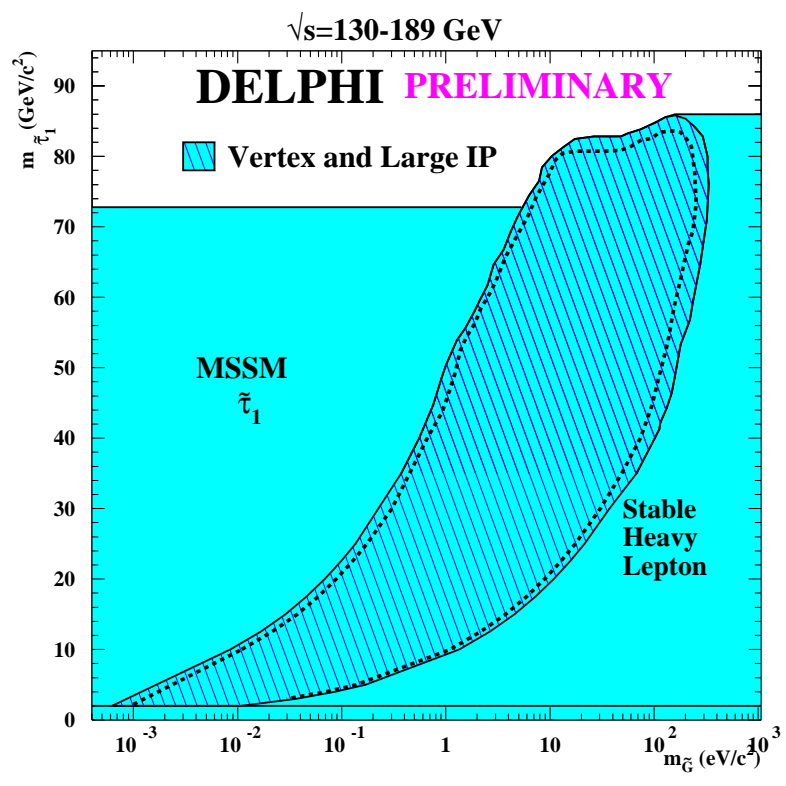

Figure 6: DELPHI lower limit on the $\widetilde{\tau}_{1}$ mass as a function of the $\widetilde{\mathrm{G}}$ mass in GMSB models, from data up to $189 \mathrm{GeV}$.

There is an ongoing effort to combine the four experiment results (on $\widetilde{\chi}_{1}^{ \pm}, \widetilde{\mathrm{e}}_{\mathrm{R}}, \widetilde{\tau}_{1}$ searches, using also Higgs searches and the $\Gamma_{Z}$ constraint) to derive an absolute limit on the $\tilde{\chi}_{1}^{0}$ mass in the mSUGRA framework. Preliminary results [26] from data up to $189 \mathrm{GeV}$ give a limit at $44 \mathrm{GeV} / c^{2}$ if the difference between the $\widetilde{\tau}_{1}$ and $\widetilde{\chi}_{1}^{0}$ masses is above $5 \mathrm{GeV} / c^{2}$ irrespective of the values of the underlying parameters, except $\mathrm{A}_{0}$ which is set to 0 . Recently, dedicated searches have been performed to cover mass differences below $5 \mathrm{GeV} / c^{2}$ and first results confirmed the validity of the above limit also in that case [27]. The next step should be to check the impact of $A_{0}$, which is expected to be large.

About $\mathrm{B}_{\mathrm{p}}$ the superpotential quoted in equation (1) is not the most complete $\mathrm{R}_{\mathrm{p}}$ violating potential. Extra bilinear terms of the form $\epsilon_{i} \mathrm{~L}_{\mathrm{i}} \mathrm{H}_{2}$, where $\mathrm{H}_{2}$ is the Higgs superfield with positive hypercharge, are also possible candidates to generate $\mathrm{B}_{\mathrm{p}}$. First results have been reported recently on a search for stops decaying through a bilinear term into a $\mathrm{b} \tau$ pair $[28]$.

Finally, if $R_{p}$ is conserved, the LSP being stable restricts the experimental sensitivity to other sparticles to mass differences between the sparticle and the LSP in excess of a few $\mathrm{GeV} / c^{2}$. Searches have been conducted to explore nearly mass degeneracy cases for the lightest chargino [29] and more recently for the lightest se- 
lectron [30]. Note that sparticles degenerated with the LSP exist in restricted regions of the parameter space of the constrained MSSM as defined in section 3.1 but they are mostly predicted in other supersymmetric scenarios, such as a constrained MSSM without gaugino mass unification at the GUT scale or the recent anomaly mediated SUSY breaking models [20].

\section{Search for extra dimensions}

It was pointed out recently [31, 20] that extra spatial dimensions, which are present in any superstring theory, can also solve the hierarchy problem, independently of the underlying theoretical framework. Indeed, if $\mathrm{n}$ extra compact spatial dimensions of radius $R$ exist, the quantum gravity scale in $n+4$ dimensions, $M_{D}$, is related to the Planck scale, $\mathrm{M}_{\mathrm{Pl}}$, by $\mathrm{M}_{\mathrm{Pl}}^{2} \sim \mathrm{R}^{n} \mathrm{M}_{\mathrm{D}}^{2+\mathrm{n}}$. If $\mathrm{R}$ and $\mathrm{n}$ are such that $\mathrm{M}_{\mathrm{D}}$ is of the order of the electroweak $(\mathrm{EW})$ scale, the hierarchy vanishes. The case $n=1$ is ruled out since it would imply quantum gravity effects observable over solar system distances.

At low energy, extra spatial dimensions are expected to manifest through the production of gravitons, G, observable in both direct searches and precise measurements. Searching for the associated production of a pair $(\gamma, G)$ in single photon final states at LEP2 leads to constraints on $\mathrm{M}_{\mathrm{D}}$ depending on $\mathrm{n}$. As an example, $\mathrm{M}_{\mathrm{D}}$ has been found to be greater than $1.1 \mathrm{TeV}, 0.7 \mathrm{TeV}$ and $0.53 \mathrm{TeV}$ for 2,4 or 6 extra dimensions, respectively [32]. Gravitons would also be responsible for deviations wrt the SM in precise measurements. Combining observables in several final states at LEP, the ultra-violet cut-off of the underlying quantum gravity theory has been constrained to be larger than $0.8 \mathrm{TeV}$ (resp. $1.1 \mathrm{TeV}$ ) if the interference between the $\mathrm{SM}$ and $\mathrm{G}$ exchange amplitudes is negative (resp. positive) [32].

\section{$5 \quad$ Higgs bosons}

The phenomenology of Higgs bosons is little model-dependent which allows to cover several theoretical frameworks with a limited number of searches. Results encompass neutral Higgs bosons as expected in the SM, MSSM and beyond, as well as charged Higgs bosons in two Higgs doublet models.

\subsection{The Standard Model Higgs boson}

In the mass range currently under study, i.e. around $100 \mathrm{GeV} / c^{2}$, only LEP would be sensitive to the SM Higgs boson. The main production process leads to pairs of Higgs and Z bosons, with the Z boson on-shell. Due to the clean experimental environment, all $\mathrm{Z}$ final states are exploited. In addition, the Higgs boson is expected to decay mainly into a $\mathrm{b} \overline{\mathrm{b}}$ pair (the branching fraction is $\sim 82 \%$ for a $100 \mathrm{GeV} / \mathrm{c}^{2}$ 
Higgs boson) so that excellent b-tagging capabilities of the LEP detectors help a lot in these searches.

\begin{tabular}{|c|c|c|c|c|c|}
\hline & bkg & data & $\begin{array}{c}\text { exp. limit } \\
\left(\mathrm{GeV} / c^{2}\right)\end{array}$ & $\begin{array}{c}\text { obs. limit } \\
\left(\mathrm{GeV} / c^{2}\right)\end{array}$ & $\begin{array}{c}\text { 1-CL } \mathrm{CL}_{b} \\
\text { at obs. lim. }\end{array}$ \\
\hline ALEPH & 44.4 & 53 & 95.9 & 92.9 & $4 \%$ \\
DELPHI & 172.7 & 187 & 94.6 & 94.1 & $20 \%$ \\
L3 & 91.1 & 94 & 94.8 & 95.3 & $64 \%$ \\
OPAL & 35.4 & 41 & 94.9 & 91.0 & $4 \%$ \\
\hline
\end{tabular}

Table 8: Rates and exclusion limits obtained at LEP in the SM Higgs boson searches in $189 \mathrm{GeV}$ data. Results observed in data are compared with expectations from background simulation. The last column gives the probability to have a less backgroundlike result than that observed.

\begin{tabular}{|c|c|c|c|c|c|}
\hline & $\begin{array}{c}\mathcal{L} \\
\left(\mathrm{pb}^{-1}\right)\end{array}$ & bkg & data & $\begin{array}{c}\text { exp. limit } \\
\left(\mathrm{GeV} / c^{2}\right)\end{array}$ & $\begin{array}{c}\text { obs. limit } \\
\left(\mathrm{GeV} / c^{2}\right)\end{array}$ \\
\hline ALEPH & 98 & 32.3 & 27 & 99.9 & 98.8 \\
DELPHI & 84 & 15.4 & 15 & 97.0 & 97.3 \\
L3 & 109 & 42.2 & 38 & 97.3 & 98.7 \\
OPAL & 85 & 21.0 & 23 & 97.3 & 95.4 \\
\hline
\end{tabular}

Table 9: Rates and exclusion limits obtained at LEP in the SM Higgs boson searches in data at 192 and $196 \mathrm{GeV}$. Results observed in data are compared with expectations from background simulation.

The results obtained at $189 \mathrm{GeV}$ by the four LEP experiments 33 are detailed in Table 8 at the level of selections where data are actually compared with simulation to test the background and signal+background hypotheses and derive exclusion limits or discovery significances. To achieve the highest sensitivity to the signal, these derivations rely on a test-statistic which, besides the rates, take also into account the pattern of the selected events (the reconstructed Higgs boson mass, $\mathrm{m}_{\mathrm{H}}$, or $\mathrm{m}_{\mathrm{H}}$ and another discriminant variable like the event b-quark content) [34]. The more information in the comparison, the earlier the event selection procedure is stopped, as illustrated in Table 8 by the different selection levels in the four experiments. There is an excess in data in three experiments, which is partly signal-like in two of them, as revealed by the difference of a few $\mathrm{GeV} / c^{2}$ between the observed and expected exclusion limits. After investigation, part of the excess was attributed to a 


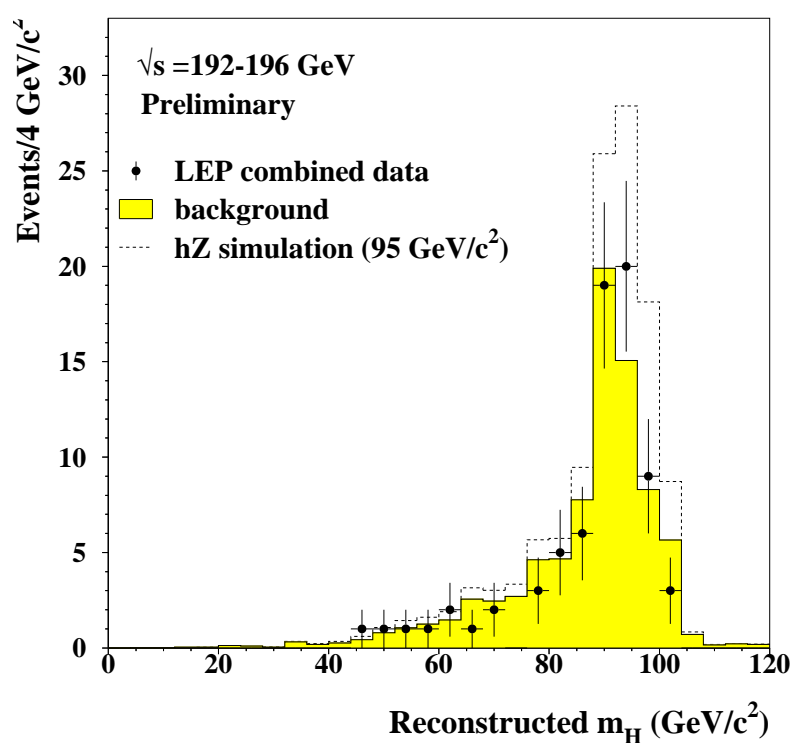

Figure 7: Reconstructed Higgs boson mass spectrum in LEP data at 192 and $196 \mathrm{GeV}$. Data (dots) are compared with background simulation (full histogram) and with signal simulation in the hypothesis of a SM Higgs boson of $95 \mathrm{GeV} / c^{2}$ (dashed histogram). 74 events are selected in data while 81.1 are expected from background.

systematic bias. When combining the four LEP experiments, the exclusion limit is $95.2 \mathrm{GeV} / c^{2}$ for an expectation of $97.2 \mathrm{GeV} / c^{2}$ [35].

A preliminary update at $196 \mathrm{GeV}$ was reported at the conference [36], as shown in Table 9. The reconstructed Higgs boson mass distribution after tighter selections is given in Figure 0. The excess seen at $189 \mathrm{GeV}$ has not been confirmed at higher energies and data agree with expectations. After the conference, these results were combined, giving an exclusion limit of $102.6 \mathrm{GeV} / c^{2}$, for an expected limit of $102.3 \mathrm{GeV} / c^{2}$ [37]. At the end of the ' 99 run, which reached $\sqrt{s}=202 \mathrm{GeV}$, experiments reported preliminary limits up to $106 \mathrm{GeV} / c^{2}$, in good agreement with the expected ones [38]. Prospects for the last run of LEP at $\sqrt{s}$ up to $206 \mathrm{GeV}$ are $114 \mathrm{GeV} / c^{2}$ for the $95 \%$ exclusion or $3 \sigma$ discovery potentials and $111 \mathrm{GeV} / c^{2}$ for the $5 \sigma$ discovery sensitivity, when the four experiments are combined [39]. Higher masses up to $180 \mathrm{GeV} / c^{2}$ should then be accessible in the future high luminosity run at the Tevatron [40]. 


\subsection{MSSM neutral Higgs bosons}

Most results about neutral Higgs bosons in the MSSM come also from LEP, which is sensitive to the two lightest bosons, $\mathrm{h}$ and $\mathrm{A}$. There are two production processes, $\mathrm{e}^{+} \mathrm{e}^{-} \rightarrow \mathrm{hZ}$, like in the SM case, and $\mathrm{e}^{+} \mathrm{e}^{-} \rightarrow \mathrm{hA}$. The two processes are complementary in the parameter space. In the mass range between 80 and $110 \mathrm{GeV} / c^{2}$, the main decay mode of both bosons is again in $b \bar{b}$ in most of the parameter space, with branching fractions greater than in the SM $(\sim 91 \%)$. The dominant hZ final states are as in the SM case, while hA is expected to give mostly b $\bar{b} b \bar{b}$ and $\tau^{+} \tau^{-} b \bar{b}$ final states. Here again, b-tagging plays a crucial rôle.

The theoretical framework of these searches is the MSSM with $R_{p}$ conservation and soft breaking terms unified at the EW scale. In the MSSM the Higgs boson masses are connected to each other, so that at tree level, there are only two free parameters: $\tan \beta$ and one Higgs boson masses, or, alternatively, two Higgs boson masses, eg $\mathrm{m}_{\mathrm{A}}$ and $\mathrm{m}_{\mathrm{h}}$. The properties of the MSSM Higgs bosons, and in particular the mass relationships, are modified by radiative corrections which introduce five additional parameters: the mass of the top quark, the Higgs mixing parameter, $\mu$, the common sfermion mass term at the $\mathrm{EW}$ scale, $\mathrm{M}_{S}$, the common $\mathrm{SU}(2)$ gaugino mass term 2 at the EW scale, $\mathrm{M}_{2}$, and the common squark tri-linear coupling at the EW scale, A. The interpretation of the experimental results depend on the values assumed for these parameters.

\subsubsection{Benchmark hypotheses}

Using leading order two-loop calculations of the radiative corrections 41], benchmark values have been defined for the parameters beyond tree-level [42]: $175 \mathrm{GeV} / \mathrm{c}^{2}$ for the top mass, $1 \mathrm{TeV} / \mathrm{c}^{2}$ for $\mathrm{M}_{S}$ and $1.6 \mathrm{TeV} / \mathrm{c}^{2}$ for $\mathrm{M}_{2}$. Two benchmark scenarios 42 have been defined for the parameters $\mathrm{A}$ and $\mu$, which determine the mixing in the stop sector: no mixing $(A=0, \mu=-100 \mathrm{GeV})$ and maximal mixing $\left(A=\sqrt{6} \mathrm{M}_{\mathrm{S}}\right.$, $\mu=-100 \mathrm{GeV}$ ). The no mixing hypothesis leads to minimal radiative corrections to $\mathrm{m}_{\mathrm{h}}$ while the maximal mixing induces the largest corrections. This defines the usual framework for the interpretation of the MSSM neutral Higgs boson searches.

Results obtained at LEP at $189 \mathrm{GeV}$ in the hA channel are summarized in Table 10. These results, together with the hZ results reinterpreted in the MSSM framework, allow to set constraints on $\mathrm{m}_{\mathrm{h}}, \mathrm{m}_{\mathrm{A}}$ and $\tan \beta$. As an example, Figure 8 represents the region of the $\left(\tan \beta, \mathrm{m}_{\mathrm{h}}\right)$ plane excluded by the combination of the LEP results up to $189 \mathrm{GeV}$, in the less favourable case of the maximal mixing [35]. Whatever the mixing hypothesis, these combined results exclude Higgs bosons up to

\footnotetext{
${ }^{2}$ The $\mathrm{U}(1)$ gaugino mass term at the $\mathrm{EW}$ scale, $\mathrm{M}_{1}$, is related to $\mathrm{M}_{2}$ through the GUT relation $\mathrm{M}_{1}=(5 / 3) \tan ^{2} \theta_{\mathrm{w}} \mathrm{M}_{2}$, while the $\mathrm{SU}(3)$ gaugino mass term, $\mathrm{M}_{3}$, is set via the gluino mass, which is taken equal to $\mathrm{M}_{S}$.
} 
$80.7 \mathrm{GeV} / c^{2}$ for $\mathrm{m}_{\mathrm{h}}$ and $80.9 \mathrm{GeV} / c^{2}$ for $\mathrm{m}_{\mathrm{A}}$ for $\tan \beta$ greater than 0.4 . At $\tan \beta \sim 1$, the experimental lower limit on $\mathrm{m}_{\mathrm{h}}$ is above the theoretical upper bound on $\mathrm{m}_{\mathrm{h}}$ so that $\tan \beta$ is excluded between 0.9 and 1.6 (0.6 and 2.6) in the maximal mixing (no mixing) hypothesis. The expected limits on both masses are $5 \mathrm{GeV} / c^{2}$ higher while the expected excluded ranges in $\tan \beta$ agree with the observed ones.

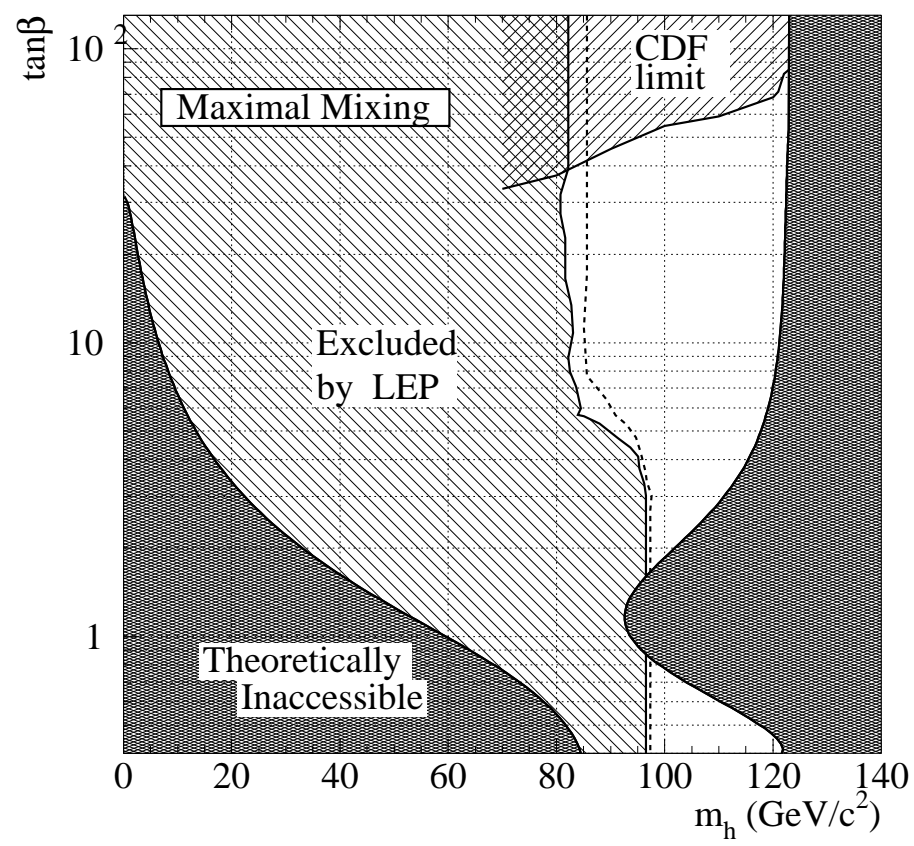

Figure 8: Regions in the $\left(\mathrm{m}_{\mathrm{h}}, \tan \beta\right)$ plane excluded by the MSSM Higgs boson searches at LEP in data up to $189 \mathrm{GeV}$, and at CDF in run I data. The regions not allowed by the MSSM for a top mass of $175 \mathrm{GeV} / \mathrm{c}^{2}$ a SUSY scale of $1 \mathrm{TeV} / \mathrm{c}^{2}$ and maximal mixing in the stop sector are also indicated. The dotted curve is the LEP expected limit.

It must be noted that, contrary to the limits on masses, the limit on $\tan \beta$ is very sensitive to the values of the underlying parameters, which have a large impact on the theoretical upper bound on $\mathrm{m}_{\mathrm{h}}$. As an example, this upper bound increases with increasing top quark masses and no limit on $\tan \beta$ is obtained if the top mass is moved by two standard deviations. The pure MSSM parameters or the order of the radiative correction calculations have also a non negligible effect. So, the excluded ranges in $\tan \beta$ cannot be taken as an absolute result, even if the maximal mixing hypothesis 


\begin{tabular}{|c|c|c|c|c|c|c|}
\hline & bkg & data & \multicolumn{2}{|c|}{$\mathrm{m}_{\mathrm{h}}$ limits $\left(\mathrm{GeV} / c^{2}\right)$} & \multicolumn{2}{c|}{$\mathrm{m}_{\mathrm{A}}$ limits $\left(\mathrm{GeV} / c^{2}\right)$} \\
& & & observed & expected & observed & expected \\
\hline ALEPH & 7.5 & 10 & 82.5 & 83.1 & 83.1 & 83.6 \\
DELPHI & 22.6 & 24 & 82.1 & 81.1 & 83.1 & 82.2 \\
L3 & 140.6 & 153 & 76.0 & 78.0 & 76.0 & 79.0 \\
OPAL & 12.9 & 15 & 74.8 & 76.4 & 76.5 & 78.2 \\
\hline
\end{tabular}

Table 10: Rates and exclusion limits obtained at LEP in the MSSM hA Higgs boson searches in $189 \mathrm{GeV}$ data. Results observed in data are compared with expectations from background simulation.

is a pessimistic scenario.

Also displayed in Figure 8 is the recent $\mathrm{CDF}$ result 43 at large $\tan \beta$. In this region, the production cross-section is large enough to make Tevatron sensitive to the production process $\mathrm{p} \overline{\mathrm{p}} \rightarrow \mathrm{b} \overline{\mathrm{b}} \mathrm{h}, \mathrm{H}, \mathrm{A}$ where one Higgs boson is emited off a bottom quark, leading to four b-tagged jets in the final state.

Results from LEP experiments with data up to $196 \mathrm{GeV}$ were also reported at the conference [36], as shown in Table 11. These results were combined later on, giving exclusion limits of $84.3 \mathrm{GeV} / c^{2}$ on $\mathrm{m}_{\mathrm{h}}$ and $84.5 \mathrm{GeV} / c^{2}$ on $\mathrm{m}_{\mathrm{A}}$, independent of the mixing hypothesis, and excluded ranges in $\tan \beta$ between 0.8 and 1.9 (0.5 and 3.2 ) in the maximal mixing (no mixing) hypothesis [37]. At the end of the '99 data taking, LEP experiments reported mass limits up to $90 \mathrm{GeV} / c^{2}$ and excluded ranges in $\tan \beta$ somewhat larger than the combined result at $196 \mathrm{GeV}$ [38]. The excess in data observed by OPAL in the hA channel at $196 \mathrm{GeV}$ was not confirmed at higher energies nor by the other experiments.

\begin{tabular}{|c|c|c|c|c|c|}
\hline & $\mathcal{L}$ & bkg & data & \multicolumn{2}{|c|}{$\mathrm{m}_{\mathrm{h}}$ limits $\left(\mathrm{GeV} / \mathrm{c}^{2}\right)$} \\
& $\left(\mathrm{pb}^{-1}\right)$ & & & observed & expected \\
\hline ALEPH & 98 & 4.8 & 1 & 85.2 & 86.1 \\
OPAL & 85 & 6.8 & 14 & 74.3 & 79.1 \\
\hline
\end{tabular}

Table 11: Rates and exclusion limits obtained at LEP in the MSSM hA Higgs boson searches in 192 and $196 \mathrm{GeV}$ data. Results observed in data are compared with expectations from background simulation. 


\subsubsection{General scans}

More general interpretations of the LEP Higgs boson searches have been performed, scanning over the MSSM underlying parameters. The parameter space is however usually restricted by imposing additional constraints, e.g. the experimental results on supersymmetric particles or the $\Gamma_{Z}$ constraint. It was shown that the benchmark limits hold in more than $99.99 \%$ of the parameter sets 44 and that the mass limits from general scans are a few $\mathrm{GeV} / c^{2}$ weaker than the benchmark ones [45]. As general scans usually vary also the top mass quark within two standard deviations, the limit on $\tan \beta$ vanishes.

\subsubsection{Recent developments}

Recent theoretical work led to two-loop calculations of the radiative corrections at the next-to-leading order and to a redefinition of the benchmark values for the underlying parameters [46]. In particular, the theoretical upper bound on $\mathrm{m}_{\mathrm{h}}$ was found to be underestimated by $\sim 7 \mathrm{GeV} / c^{2}$ in the maximal mixing scenario previously used. The new scenario now proposed (called $\mathrm{m}_{\mathrm{h}}{ }^{\max }$ scenario) should lead to more realistic bounds on $\tan \beta$. Another new scenario (called large $\mu$ scenario) with the $\mathrm{h}$ boson within kinematical reach at LEP but with vanishing branching fraction into $\mathrm{b} \overline{\mathrm{b}}$ was proposed to check the sensitivity of LEP to Higgs bosons with non-dominant b decays. Future LEP results will be derived in these new benchmark schemes.

\subsection{Neutral Higgs bosons beyond MSSM}

Searches for neutral Higgs bosons as expected beyond the MSSM have also been performed, mostly at LEP. Three lines of searches have been followed. First, the existing LEP analyses on MSSM h and A bosons have been used, either as such or with some modifications (e.g. relaxing the b-tagging requirements) to cover the final states expected in more general models. Thus, a first study showed that LEP is sensitive to neutral Higgs bosons of two Higgs doublet models (2HDM), even in a scenario with dominant decays into $c \bar{c}$ or in a model with $\mathrm{CP}$ violation [47]. Recently, the 2HDM parameter space (with CP conservation) has been explored in a detailed scan 48. Finally, for the first time, a non minimal supersymmetric model containing one gauge-singlet Higgs field in addition to the MSSM has also been investigated 49.

As a second research line, the case of a Higgs boson $h$ decaying invisibly has been studied. Dedicated searches in the hZ channel translate into upper limits on the production cross-section times branching ratio, which are compared with expectations from specific models [50]. As an example, assuming a SM production rate and a 100\% branching ratio into invisible products, a lower limit on $\mathrm{m}_{\mathrm{h}}$ at $95.4 \mathrm{GeV} / c^{2}$ is obtained.

The third topic deals with a Higgs boson h with anomalous couplings to photons. From dedicated searches in the hZ, h $\gamma$ and hA channels, general constraints are set 
on the production cross-section times branching ratio or directly on the anomalous couplings. They are again compared with expectations from specific models [51, 36]. As an example, assuming a SM production rate and a fermiophobic Higgs boson, a lower limit on $\mathrm{m}_{\mathrm{h}}$ at $97.5 \mathrm{GeV} / c^{2}$ is achieved.

\subsection{Charged Higgs bosons}

Recent results on charged Higgs bosons, $\mathrm{H}^{ \pm}$, have been reported by the LEP experiments. The framework of these searches is the general 2HDM scheme with as sole free parameters the $\mathrm{H}^{ \pm}$mass and its leptonic decay branching fraction, assuming that the hadronic (into cs) and leptonic (into $\tau \nu_{\nu}$ ) decays saturate the width of the particle, which is the case in the mass range below $m_{W}$ that is presently tested.

\begin{tabular}{|c|c|c|c|c|}
\hline & bkg & data & $\begin{array}{c}\text { expected limit } \\
\left(\mathrm{GeV} / c^{2}\right)\end{array}$ & $\begin{array}{c}\text { observed limit } \\
\left(\mathrm{GeV} / c^{2}\right)\end{array}$ \\
\hline ALEPH & 333.5 & 302 & 69.5 & 65.5 \\
DELPHI & 213.0 & 215 & 66.5 & 66.9 \\
L3 & 523.5 & 499 & 71.2 & 67.5 \\
OPAL & 241.1 & 252 & 68.5 & 68.7 \\
\hline
\end{tabular}

Table 12: Rates and exclusion limits obtained at LEP in the charged Higgs boson searches in $189 \mathrm{GeV}$ data. Results observed in data are compared with expectations from background simulation.

The results obtained by the LEP experiments at $189 \mathrm{GeV}$ 52 are shown in Table 12. Once combined, these results exclude an $\mathrm{H}^{ \pm}$boson up to $77.3 \mathrm{GeV} / c^{2}$ whatever its leptonic decay branching ratio, while the expected limit is $74.9 \mathrm{GeV} / c^{2}$ 3.5. Results from data up to $196 \mathrm{GeV}$ were combined after the conference, with no improvement in the mass limit independent of the branching ratio, due to the large background from WW pairs which is penalizing for the analyses in the hadronic mode. On the other hand, $\mathrm{H}^{ \pm}$bosons with pure $(50 \%)$ leptonic decays have been excluded up to $84.9(78.4) \mathrm{GeV} / c^{2}$ by the same results [37]. Individual limits reported at the end of the '99 data taking were below the combined results at $196 \mathrm{GeV}$ [38.

Higher masses were tested at the Tevatron runI, searching for $\mathrm{H}^{ \pm}$in decays of pair-produced top quarks. The mass limits are $\tan \beta$ dependent and restricted to those values of $\tan \beta$ for which the top quark branching fraction into $\mathrm{H}^{+} \mathrm{b}$ is large enough. In the most favourable case $(\tan \beta=150)$ masses up to $153 \mathrm{GeV} / c^{2}$ have been excluded [53]. 


\section{Conclusions}

New particle searches cover an impressive variety of topics and topologies. The way results are interpreted has undergone substantial changes during the past few years. To get higher sensitivity to the researched signals, different channels and/or experiments are combined and more information is put in the statistical analysis of the results, like in the Higgs boson searches. There is also an effort to go to more modelindependent results by relaxing theoretical assumptions, scanning parameter values or testing more general models. Supersymmetric particle searches are examples of that kind.

To give but a few results, in gravity-mediated SUSY breaking models, the lightest neutralino has been excluded up to $32 \mathrm{GeV} / c^{2}$ whether $\mathrm{R}_{\mathrm{p}}$ is conserved or not, while the lightest chargino has been excluded up to 68 (94) $\mathrm{GeV} / c^{2}$ if $\mathrm{R}_{\mathrm{p}}$ is conserved (broken). A SM Higgs boson has been excluded up to $106 \mathrm{GeV} / c^{2}$, MSSM neutral Higgs bosons up to $90 \mathrm{GeV} / c^{2}$ and $2 \mathrm{HDM}$ charged Higgs bosons up to $77 \mathrm{GeV} / c^{2}$.

I am grateful to all my colleagues who provided me with information when preparing this talk. I would like to thank M.Besancon, R.Nikolaidou, E.Perez and D.Treille for very helpful discussions.

\section{References}

[1] CDF Coll., Phys. Rev. Lett. 83, 3124 (1999) and FERMILAB-PUB-99/141-E, subm. to Phys. Rev. Lett.; L3 Note 2428.

[2] CDF Coll., Phys. Rev. Lett. 79, 2191 (1997); OPAL Physics note PN372; L3 Note 2425.

[3] ALEPH 99-018 CONF 99-013; DELPHI 99-135 CONF 322; L3 Note 2402; OPAL Coll., CERN-EP/99-097, acc. by Eur. Phys. J. C .

[4] CDF Coll., Phys. Rev. Lett. 79, 2198 (1997); DO Coll., Phys. Rev. Lett. 82, 2457 (1999) and Phys. Rev. Lett. 82, 4769 (1999); H1 contribution to EPSHEP99, abstract 157f; ZEUS Coll., DESY 99-058, subm. to Eur. Phys. J. C; OPAL Physics note PN409.

[5] CDF Coll., Phys. Rev. D 55, R5263 (55) 1997; DO Coll., contribution to the XVIII Lepton Photon Symposium, July 1997; H1 contribution to ICHEP98, abstract 581; ZEUS contribution to EPS-HEP99, abstract 555; ALEPH 99-068 CONF 99-042 and 99-049 CONF 99-025; DELPHI 99-71 CONF 258 and 99-137 CONF 324; L3 Coll., CERN-EP/99-138, acc. by Phys. Lett. B; OPAL Physics Note PN413. 
[6] W. Buchmüller, R. Rückl and D. Wyler, Phys. Lett. B 191, 442 (1987) and Erratum Phys. Lett. B 448, 320 (1999).

[7] H1 Coll., DESY-99-081, acc. by Eur. Phys. J. C.

[8] S. Hagopian, 'Leptoquark summary from the Tevatron, CDF and DO', in Proc. of 13th Topical Conference on Hadron Collider Physics (HCP99), Mumbai, India (January 14-20, 1999) and ref. therein; ZEUS contribution to ICHEP98, abstract 754 and contributions to EPS-HEP99, abstracts 546 and 552; ALEPH 99-018 CONF 99-013; DELPHI 99-80 CONF 267; L3 Note 2402.

[9] LEPSUSYWG, ALEPH, DELPHI, L3 and OPAL experiments, note LEPSUSYWG/99-01.1 and note LEPSUSYWG/99-02.1.

[10] ALEPH 99-78 CONF 99-50; DELPHI 99-95 CONF 282; L3 note 2444 (1999); OPAL Physics note PN413.

[11] CDF Coll., FERMILAB-PUB-99/311-E, subm. to Phys. Rev. Lett., and FERMILAB-PUB-99/340-E, subm. to Phys. Rev. Lett..

[12] DO Coll., Phys. Rev. Lett. 83, 4937 (1999).

[13] ALEPH 99-11 CONF 99-6; DELPHI 99-82 CONF 269 and 99-88 CONF 275.

[14] L3 Coll., CERN-EP/99-127, subm. to Phys. Lett. B; OPAL Coll., CERN-EP/99123, subm. to Phys. Lett. B.

[15] ALEPH 99-012 CONF 99-007; DELPHI 99-79 CONF 266, 99-93 CONF 280 and 99-96 CONF 283; OPAL Physics notes PN394 and PN411; L3 Note 2410.

[16] ALEPH 99-018 CONF 99-013; DELPHI 99-135 CONF 322; L3 Note 2399; OPAL Coll., CERN-EP/99-097, acc. by Eur. Phys J. C.

[17] ALEPH 99-018 CONF 99-013; L3 Note 2402.

[18] H1 contribution to ICHEP98, abstract 580; ZEUS contribution to EPS-HEP99, abstract 548 .

[19] CDF Coll., Phys. Rev. Lett. 83, 2133 (1999); DO Coll., Phys. Rev. Lett. 83, 4476 (1999); D.R. Claes, 'CDF/DO search for new phenomena II', in Proc. of the XXXIVth Rencontres de Moriond, Electroweak interactions and Unified Theories, Les Arcs, France, (March 13-20, 1999).

[20] G. Giudice, these Proceedings. 
[21] ALEPH 99-049 CONF 99-025; DELPHI 99-77 CONF 264; L3 Note 2426 (1999); OPAL Physics Note PN386; LEPSUSYWG, note LEPSUSYWG/99-04.2 and note LEPSUSYWG/99-05.2.

[22] CDF Coll., Phys. Rev. Lett. 81, 1791 (1998); DO Coll., Phys. Rev. Lett. 80, 442 (1998).

[23] A. Castro, 'Light gravitino search at CDF', in Proc. of the 6th International Conference on Supersymmetries in Physics (SUSY99), Fermilab (June 1999).

[24] ALEPH Coll., CERN-EP/99-171, subm. to Eur. Phys J. C; DELPHI 99-82 CONF269, 99-89 CONF 276 and 99-90 CONF 277; L3 Note 2433; OPAL Physics Note PN397.

[25] ALEPH Coll., CERN-EP/99-171, subm. to Eur. Phys J. C.

[26] G. Ganis, 'LEP SUSY working group status report', LEPC meeting, CERN, September 7, 1999 and LEPSUSYWG, note LEPSUSYWG/99-03.1.

[27] J. Marco, 'DELPHI status report', LEPC meeting, CERN, November 9, 1999.

[28] L3 Note 2410.

[29] DELPHI 99-84 CONF 271; L3 Note 2431.

[30] L3 Coll., CERN-EP/99-128, acc. by Phys. Lett. B.

[31] N. Arkani-Hamed, S. Dimopoulos and G. Dvali, SLAC-PUB-7769 and SLACPUB-7864 (1999).

[32] ALEPH 99-051 CONF 99-027; DELPHI 99-77 CONF 264, 99-137 CONF 324 and 99-135 CONF 322; OPAL Physics notes PN386, PN409 and PN381; L3 Coll., CERN-EP/99-146, acc. by Phys. Lett. B.

[33] ALEPH 99-053 CONF 99-029; DELPHI 99-86 CONF 273; L3 Coll., Phys. Lett. B 461, 376 (1999) and CERN-EP/99-145, acc. by Phys. Lett. B; OPAL Coll., CERN-EP/99-096, acc. by Eur. Phys. J. C.

[34] LEPHIGGSWG, ALEPH, DELPHI, L3 and OPAL collaborations, CERNEP/98-046 and CERN-EP/99-060.

[35] LEPHIGGSWG, ALEPH, DELPHI, L3 and OPAL collaborations, ALEPH 99081 CONF 99-052 or DELPHI 99-142 CONF 327 or L3 note 2442 or OPAL Technical note TN614. 
[36] ALEPH 99-084 CONF 99-053; OPAL Physics Note PN414; DELPHI and L3, private communication.

[37] P. McNamara, 'LEP HIGGS working group status report', LEPC meeting, CERN, September 7, 1999 and http://www.cern.ch/LEPHIGGS/talks.

[38] A. Blondel (ALEPH), J.Marco (DELPHI), G. Rahal-Callot (L3) and P. Ward (OPAL), status report from the four LEP experiments, LEPC meeting, CERN, November 9, 1999.

[39] E. Gross and A. Read, 'Prospects for SM Higgs in 2000 at LEP', Pre-Chamonix meeting, CERN, January 14, 2000.

[40] J.S. Conway, 'Higgs searches in run2 at the Tevatron', in Proc. of the 13th Rencontres de Physique de la Valle d'Aoste, La Thuile, Italy (February 28-March 6, 1999).

[41] M. Carena, M. Quiros and C. Wagner, Nucl. Phys. B461 (1996) 407.

[42] Physics at LEP2, ed. G. Altarelli, T. Sjöstrand and F. Zwirner, CERN Report 96-01, Vol. 1, p. 399.

[43] J.A. Valls, CDF Note 4641 and FERMILAB-CONF-99/263-E.

[44] ALEPH Coll., Phys. Lett. B 440, 1998 (419).

[45] DELPHI 99-73 CONF 260; OPAL Coll., CERN-EP/99-096, acc. by Eur. Phys. J. C.

[46] S. Heinemeyer, W. Hollik and G. Weiglein, DESY 99-120 or hep-ph/99-060; M. Carena, S. Heinemeyer, C.E.M. Wagner and G. Weiglein, CERN-TH/99-374.

[47] DELPHI 99-86 CONF 273.

[48] OPAL Physics Note PN416.

[49] DELPHI 99-97 CONF 284.

[50] ALEPH Coll., Phys. Lett. B 466, 1999 (50); DELPHI 99-83 CONF 270; L3 Note 2435; OPAL Physics Note PN399.

[51] DO Coll., Phys. Rev. Lett. 82, 1999 (2244); ALEPH 99-056 CONF 99-030; DELPHI 99-72 CONF 259; L3 Notes 2429 and 2430; OPAL Coll., Phys. Lett. B 464, 1999 (311).

[52] ALEPH 99-070 CONF 99-044; DELPHI 99-92 CONF 279; L3 Coll., Phys. Lett. B 466, 1999 (71); OPAL Physics Note PN373. 
[53] DO Coll., Phys. Rev. Lett. 82, 1999 (4975).

\section{Discussion}

Lee Roberts (Boston University): Can you comment again on the limits on $\tan \beta$ from the Higgs searches?

Ruhlmann-Kleider: The limits on $\tan \beta$ have been derived with two-loop leading order calculations of the radiative corrections and for specific values of the underlying parameters so they cannot be regarded as absolute, even if the maximal mixing scenario represents a difficult case. Going to next-to-leading order two-loop calculations, varying the underlying parameters or increasing the top quark mass will make the excluded range in $\tan \beta$ decrease if not vanish. 